\title{
Laser Spectroscopy of Muonic Atoms and Ions
}

Randolf Pohl, ${ }^{1,2}$ François Nez, ${ }^{3}$ Luis M. P. Fernandes, ${ }^{4}$ Marwan Abdou Ahmed, ${ }^{5}$ Fernando D. Amaro, ${ }^{4}$ Pedro Amaro, ${ }^{6}$ François Biraben, ${ }^{3}$ João M. R. Cardoso, ${ }^{4}$ Daniel S. Covita, ${ }^{7}$ Andreas Dax,${ }^{8,9}$ Satish Dhawan, ${ }^{8}$ Marc Diepold, ${ }^{1}$ Beatrice Franke, ${ }^{1}$ Sandrine Galtier ${ }^{3}$ Adolf Giesen,,${ }^{5,10, \dagger}$ Andrea L. Gouvea,${ }^{4}$ Johannes Götzfried, ${ }^{1}$ Thomas Graf, ${ }^{5}$ Theodor W. Hänsch, ${ }^{1, \uparrow}$ Malte Hildebrandt, ${ }^{9}$ Paul Indelicato, ${ }^{3}$ Lucile Julien, ${ }^{3}$ Klaus Kirch, ${ }^{11,9}$ Andreas Knecht, ${ }^{9}$ Paul Knowles, ${ }^{12,8}$ Franz Kottmann, ${ }^{11}$ Julian J. Krauth, ${ }^{1}$ Eric-Olivier Le Bigot, ${ }^{3}$ Yi-Wei Liu, ${ }^{13}$ José A. M. Lopes, ${ }^{4, \#}$ Livia Ludhova, ${ }^{12, \diamond}$ Jorge Machado, ${ }^{6}$ Cristina M. B. MonteIro, ${ }^{4}$ Françoise Mulhauser, ${ }^{12,1, \odot}$ Tobias Nebel, ${ }^{1}$ Paul Rabinowitz, ${ }^{14}$ Joaquim M. F. dos Santos, ${ }^{4}$ José Paulo Santos, ${ }^{6}$ Lukas A. Schaller, ${ }^{12}$ Karsten Schummann, ${ }^{11,10,9}$ Catherine Schwob, ${ }^{3}$ Csilla I. Szabo, David TaqQu, ${ }^{9}$ João F. C. A. Veloso, ${ }^{7}$ and Andreas Voss,${ }^{5}$ Birgit Weichelt, ${ }^{5}$ Aldo ANTOGNINI $1,11,9$

The CREMA Collaboration

${ }^{1}$ Max-Planck-Institut für Quantenoptik, 85748 Garching, Germany.

${ }^{2}$ Johannes Gutenberg-Universität Mainz, QUANTUM, Institut für Physik E Exzellenzcluster PRISMA, 55099 Mainz, Germany.

${ }^{3}$ Laboratoire Kastler Brossel, UPMC-Sorbonne Universités, CNRS, ENS-PSL Research University, Collège de France, 75005 Paris, France.

${ }^{4}$ LIBPhys, Department of Physics, University of Coimbra, 3004-516 Coimbra, Portugal.

${ }^{5}$ Institut für Strahlwerkzeuge, Universität Stuttgart, - 70569 Stuttgart, Germany.

${ }^{6}$ Laboratório de Instrumentação, Engenharia Biomédica e Física da Radiação

(LIBPhys-UNL), Departamento de Física, Faculdade de Ciências e Tecnologia, FCT, Universidade Nova de Lisboa, 2829-516 Caparica, Portugal.

${ }^{7}$ I3N, Departamento de Física, Universidade de Aveiro, 3810-193 Aveiro, Portugal.

${ }^{8}$ Physics Department, Yale University, New Haven, CT 06520-8121, USA.

${ }^{9}$ Paul Scherrer Institute, 5232 Villigen-PSI, Switzerland.

${ }^{10}$ Dausinger $\mathcal{F}$ Giesen GmbH, Rotebühlstr. 87, 70178 Stuttgart, Germany.

${ }^{11}$ Institute for Particle Physics, ETH Zurich, 8093 Zurich, Switzerland.

${ }^{12}$ Département de Physique, Université de Fribourg, 1700 Fribourg, Switzerland.

${ }^{13}$ Physics Department, National Tsing Hua University, Hsinchu 300, Taiwan.

${ }^{14}$ Department of Chemistry, Princeton University, Princeton, NJ08544-1009, USA.

${ }^{\dagger}$ present address: Deutsches Zentrum für Luft- und Raumfahrt e.V. in der Helmholtz-Gemeinschaft, 70569 Stuttgart, Germany.

- also at Ludwig-Maximilians-Universität, Munich, Germany.

$\S$ present address: LogrusData, Vienna, Austria.

\# also at Instituto Politécnico de Coimbra, ISEC, 3030-199, Portugal.

$\diamond$ present address: Forschungzentrum Jülich IKP-2 and RWTH Aachen University, Germany.

$\odot$ present address: IAEA, Vienna, Austria.

\# present address: Theiss Research Inc. La Jolla, CA, USA.

E-mail:pohl@uni-mainz.de

(Received Sept. 4, 2016) 
Laser spectroscopy of the Lamb shift (2S-2P energy difference) in light muonic atoms or ions, in which one negative muon $\mu^{-}$is bound to a nucleus, has been performed. The measurements yield significantly improved values of the root-mean-square charge radii of the nuclei, owing to the large muon mass, which results in a vastly increased muon wave function overlap with the nucleus. The values of the proton and deuteron radii are 10 and 3 times more accurate than the respective CODATA values, but 7 standard deviations smaller. Data on muonic helium-3 and -4 ions is being analyzed and will give new insights. In future, the (magnetic) Zemach radii of the proton and the helium-3 nuclei will be determined from laser spectroscopy of the $1 \mathrm{~S}$ hyperfine splittings, and the Lamb shifts of muonic $\mathrm{Li}, \mathrm{Be}$ and $\mathrm{B}$ can be used to improve the respective charge radii.

KEYWORDS: laser spectroscopy, Lamb shift, muonic atoms, proton, deuteron, charge radius

\section{Introduction}

When negative muons $\left(\mu^{-}\right)$are brought to rest in matter, they can form a muonic atom or ion by ejecting all of the atom's electrons [1]. Thus, a single muon is bound to a nucleus. The muonic atom/ion will quickly deexcite, mostly ending in the $1 \mathrm{~S}$ ground state. A few per cent of the muons can reach the metastable $2 \mathrm{~S}$ state, however. For light atoms, hydrogen to boron, the metastable $2 \mathrm{~S}$ state can have a lifetime of microseconds to tens of nanoseconds [2-4] that may make these metastable $2 \mathrm{~S}$ atoms susceptible to laser spectroscopy $[5,6]$.

For an isolated muonic atom/ion, the $2 \mathrm{~S}$ lifetime is limited by the muon lifetime of $2.2 \mu \mathrm{s}$, twophoton decay to the $1 \mathrm{~S}$ ground state, and nuclear muon capture, which is still small for light muonic ions [7].

In a gaseous environment like $\mathrm{H}_{2}, \mathrm{D}_{2}, \mathrm{He}$, etc., collisional effects may shorten the $2 \mathrm{~S}$ lifetime [8]. Thus, our recent experiments in muonic hydrogen [9,10], muonic deuterium [11] and muonic helium ions $[12,13]$ were performed at gas pressures around $1 \mathrm{hPa}$, where the $2 \mathrm{~S}$ lifetimes are on the order of $1 \mu \mathrm{s}[3,14]$.

(a) muonic hydrogen, $\mu \mathrm{p}$

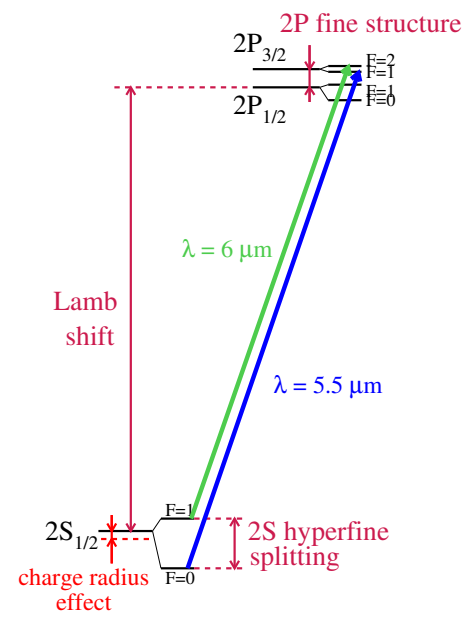

(b) muonic deuterium, $\mu \mathrm{d}$

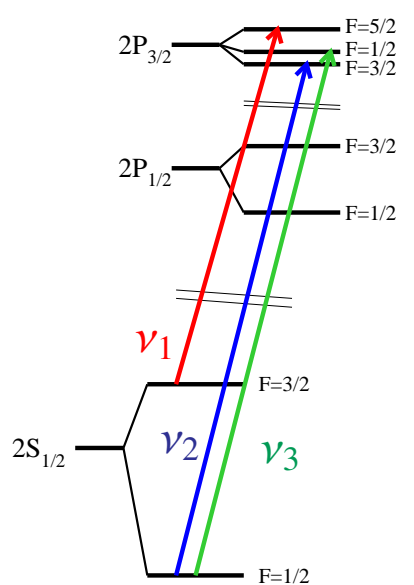

(c) muonic helium-4, $\left(\mu^{4} \mathrm{He}\right)^{+}$

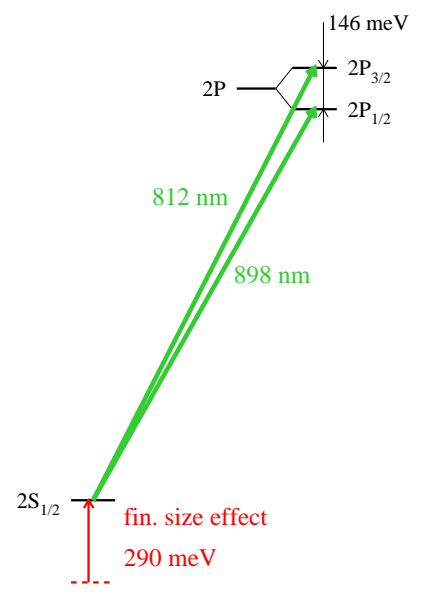

Fig. 1. Sketch of the $n=2$ levels in muonic hydrogen (left) and deuterium (center, not to scale) and muonic helium-4 ions (right). The nuclear charge radius shifts the $2 \mathrm{~S}$ level upwards, as indicated for muonic hydrogen. The measured transitions are indicated. Muonic helium-3 ions, which were also measured, are not shown here.

The principle of the measurement is the to excite the $2 S \rightarrow 2 P$ Lamb shift transition by a tunable 

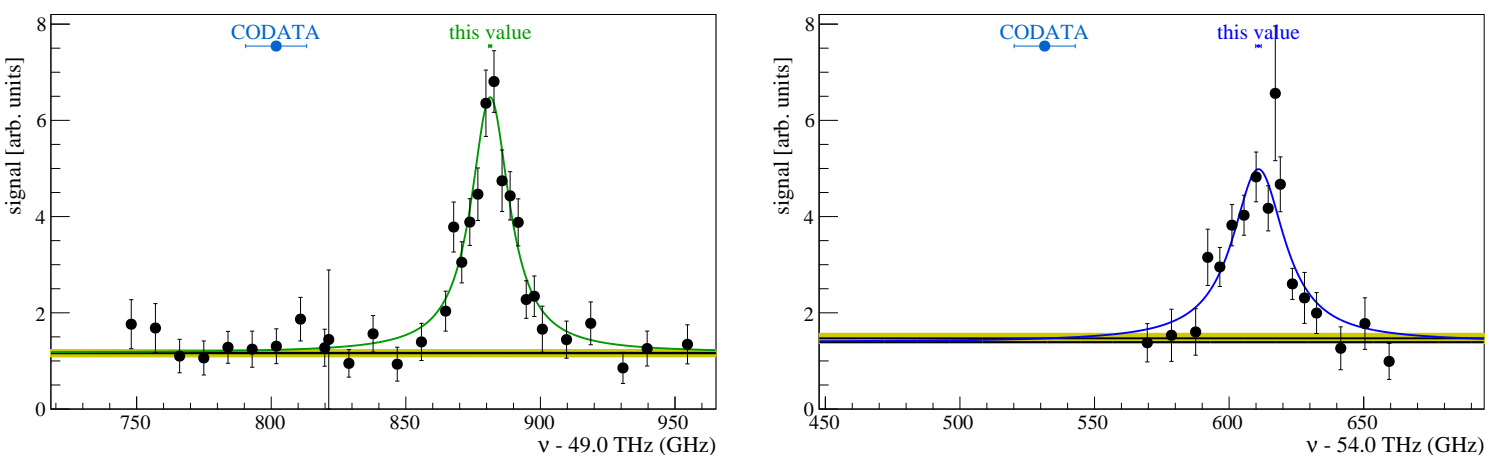

Fig. 2. Two resonances measured in $\mu \mathrm{p}$ see Fig. 1(a). Left: $2 S_{1 / 2}^{F=1}-2 P_{3 / 2}^{F=2}$ transition from the $\mathrm{F}=1$ triplet state near $\lambda=6.0 \mu \mathrm{m}(49881.35 \pm 0.65 \mathrm{GHz})$. Right: The $2 S_{1 / 2}^{F=0}-2 P_{3 / 2}^{F=1}$ transition from the $\mathrm{F}=0$ singlet state near $\lambda=5.5 \mu \mathrm{m}(54611.16 \pm 1.03 \mathrm{GHz})$. The horizontal bar indicates the background level (with uncertainty), including data taken without laser. The expected resonance positions calculated using the CODATA value of $r_{\mathrm{E}}(\mathrm{p})$ are $80 \mathrm{GHz}$ below the observed positions.

pulsed laser system $[15,16]$ and record the Lyman- $\alpha$ x-rays [17-19] from the resulting $2 P \rightarrow 1 S$ deexcitation.

The laser frequency of the $2 \mathrm{~S}-2 \mathrm{P}$ transition in light muonic atoms can then be used to determine accurate values of nuclear charge radii or polarizabilities [20-22].

\section{Lamb shift measurements and the charge radii}

\subsection{Proton radius from muonic hydrogen}

The Lamb shift in muonic hydrogen atoms, $\mu$ p, was first successfully measured at PSI in 2009. A few unsuccessful searches were performed in the preceding years $[23,24]$ at the wrong laser wavelengths, because the expected resonance positions were based on a too large proton charge radius. Finally, two transitions were measured $[9,10]$. Using the most recent theory from many authors [25-37], summarized in Ref. [38], we were able to deduce a value of the proton rms charge radius $r_{\mathrm{E}}(p)=0.84087(39) \mathrm{fm}$. This value is $7 \sigma$ smaller than the 2010-CODATA value [39], see Fig. 2. This discrepancy is now known as the "proton radius puzzle" [40,41].

It is interesting to note that the muonic hydrogen value is smaller than both the value from spectroscopy of regular hydrogen alone [42] and the widely accepted analyses of elastic electron-proton scattering data [43-47]. However, several analyses of e-p scattering data have found radii that are in agreement with the muonic hydrogen value [48-53].

\subsection{Deuteron radius from muonic deuterium}

Very recently, theory calculations in muonic deuterium [35,54-57], and in particular the difficult deuteron polarizability effects [58-62] became accurate enough to determine a deuteron charge radius from our measurements in muonic deuterium. Again, we summarized these earlier works [63]. The $2 S$ Lamb shift in muonic deuterium is

$$
\Delta E_{\mathrm{LS}}=228.7766(10) \mathrm{meV}+\Delta E_{\mathrm{LS}}^{\mathrm{TPE}}-6.1103(3) r_{\mathrm{E}}(\mathrm{d})^{2} \mathrm{meV} / \mathrm{fm}^{2} .
$$

Using the calculated contribution from two-photon exchange, also known as the deuteron polarizability contribution

$$
\Delta E_{\mathrm{LS}}^{\mathrm{TPE}}(\text { theo })=1.7096(200) \mathrm{meV}
$$

we determine the deuteron rms charge radius from a measurement of three $2 \mathrm{~S}-2 \mathrm{P}$ transitions in 

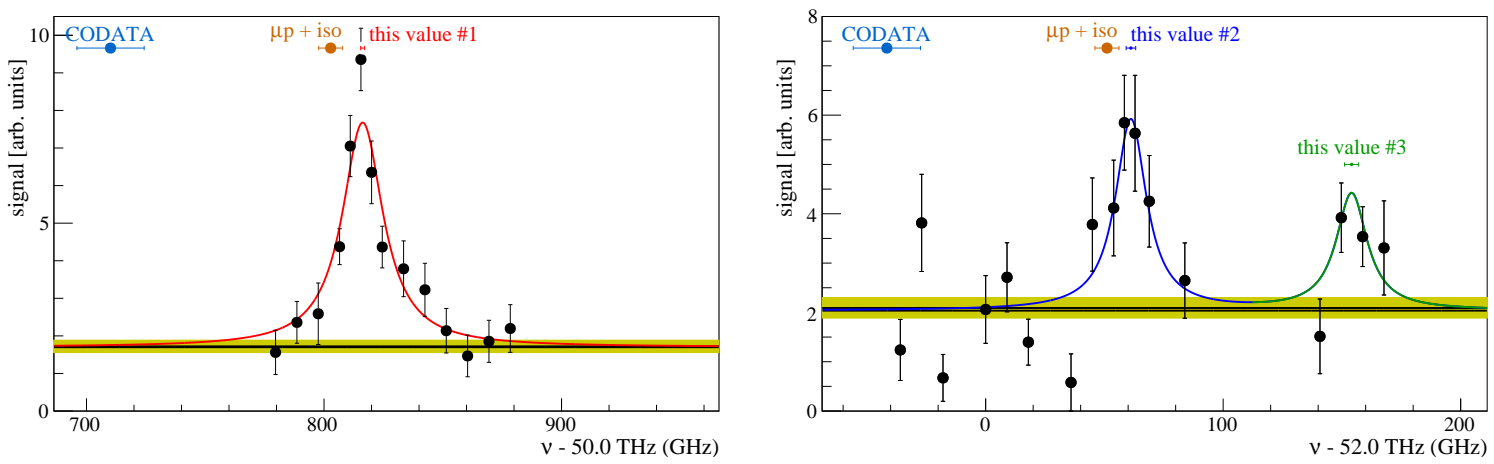

Fig. 3. Three resonances measured in $\mu \mathrm{d}$ see Fig. 1(b). Left: $2 S_{1 / 2}^{F=3 / 2} \rightarrow 2 P_{3 / 2}^{F=5 / 2}$, and right: $2 S_{1 / 2}^{F=1 / 2} \rightarrow$ $2 P_{3 / 2}^{F=3 / 2}$, and $2 S_{1 / 2}^{F=1 / 2} \rightarrow 2 P_{3 / 2}^{F=1 / 2}$. The colors are the same as in Fig. 1(b). The horizontal bar indicates the background level (with uncertainty), including data taken without laser. The expected resonance positions calculated using the CODATA value of $r_{\mathrm{E}}(\mathrm{d})$ are $100 \mathrm{GHz}$ below the observed positions. In contrast, the proton radius $r_{\mathrm{E}}(\mathrm{p})$ from muonic hydrogen, when combined with the electronic isotope shift, yields a prediction of the resonance position " $\mu \mathrm{p}+$ iso" which is within $2.6 \sigma$ of the observed ones. The muonic proton and deuteron radii are hence compatible.

muonic deuterium [11]

$$
r_{\mathrm{E}}(\mathrm{d})[\mu \mathrm{d}]=2.12562(13)_{\exp }(77)_{\text {theo }} \mathrm{fm} \text {. }
$$

Similar to the proton radius puzzle, this value from muonic deuterium is $7 \sigma$ smaller than the CODATA2010 deuteron charge radius, see Fig. 4.

We can also determine a value of the deuteron charge radius $r_{\mathrm{E}}(\mathrm{d})[\mu \mathrm{p}+\mathrm{iso}]=2.12771(22) \mathrm{fm}$ from combining the electronic isotope shift of the 1S-2S transition in H and D [64, 65],

$$
r_{\mathrm{E}}(\mathrm{d})^{2}-r_{\mathrm{E}}(\mathrm{p})^{2}=3.82007(65) \mathrm{fm}^{2}
$$

and the proton charge radius from muonic hydrogen [10]. Using this value in Eq. (1), and the measured Lamb shift in muonic deuterium, we can determine the deuteron polarizability from experiment

$$
\Delta E_{\mathrm{LS}}^{\mathrm{TPE}}(\exp )=1.7638(68) \mathrm{meV}
$$

which is three times more accurate than the theoretical value [63], and thus a benchmark for future high-precision calculations.

\subsection{Muonic helium ions}

In two beam times in 2013 and 2014 we have measured several transition frequencies in muonic helium-3 and -4 ions $[12,13]$. An example of a measured resonance is given in Fig. 5. Again, by comparing the theoretical prediction of many authors [33-35, 66-73], as summarized by us [74], values of the alpha-particle and helion charge radii can be deduced with an accuracy of about $3 \times 10^{-4}$. These will be compared with the values from electron scattering $[75,76]$ and He spectroscopy $[77-$ 83].

Besides providing important information useful for the resolution of the proton radius puzzle, these radii are interesting parameters to be compared with scattering results [75,76], few-nucleon abinitio calculations [84], and effective nuclear theories [73, 85-87]. Moreover, the muonic radii open the way to enhanced bound-state QED tests for one- and two-electron systems from measurements in "regular" $\mathrm{He}$ and $\mathrm{He}^{+}$ions $[12,77-82,88]$. They will also be used to disentangle the $4 \sigma$ discrepancy between several ${ }^{3} \mathrm{He}-{ }^{4} \mathrm{He}$ isotopic shifts measurements [77-80], and serve as anchor points to provide absolute radii for ${ }^{6} \mathrm{He}$ and ${ }^{8} \mathrm{He}$ halo nuclei when combined with the corresponding isotopic shifts measurements [85]. 

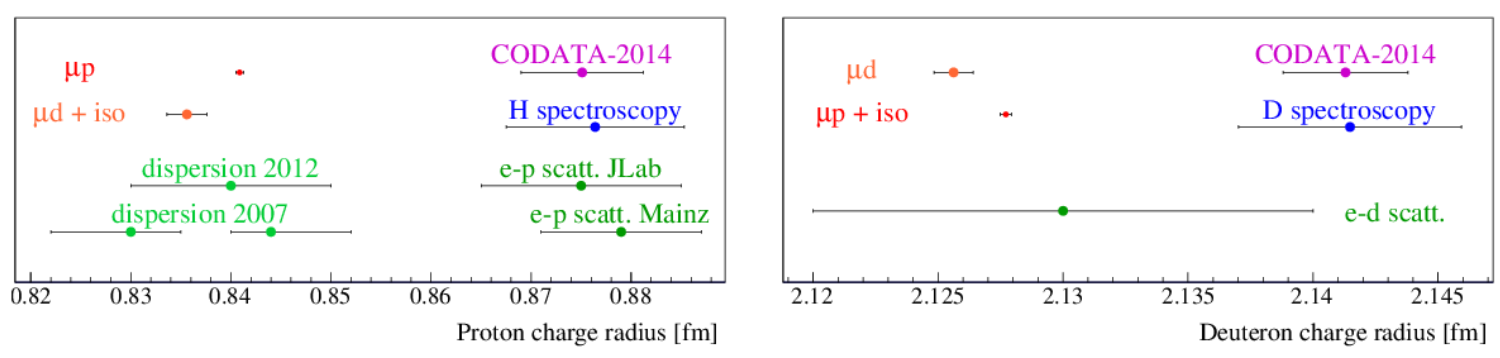

Fig. 4. Root-mean-square charge radii of the proton (left) and deuteron (right) from various sources. The muonic values are within $2.6 \sigma$ compatible with each other, when considering the $1 \mathrm{~S}-2 \mathrm{~S}$ isotope shift in regular hydrogen and deuterium $[64,65]$. Values "H spectroscopy" and "D spectroscopy" are uncorrelated, as explained in [42]. The discrepancy between muonic and electronic deuterium is hence a new discrepancy.

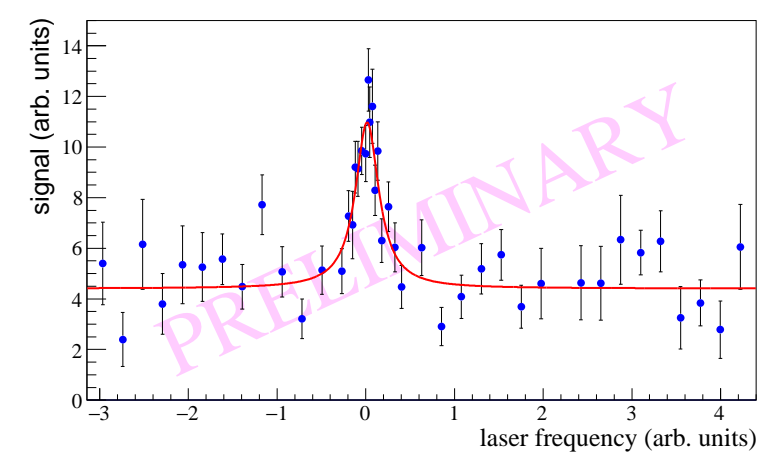

Fig. 5. Preliminary resonance plot of the 1 st muonic ${ }^{4} \mathrm{He}$ resonance (online data). The alpha particle charge radius extracted from this resonance will be about five times more accurate than the one from elastic electron scattering. The muonic charge radius uncertainty is completely dominated by the accuracy of the nuclear polarizability.

\section{Hyperfine splitting of muonic hydrogen and helium}

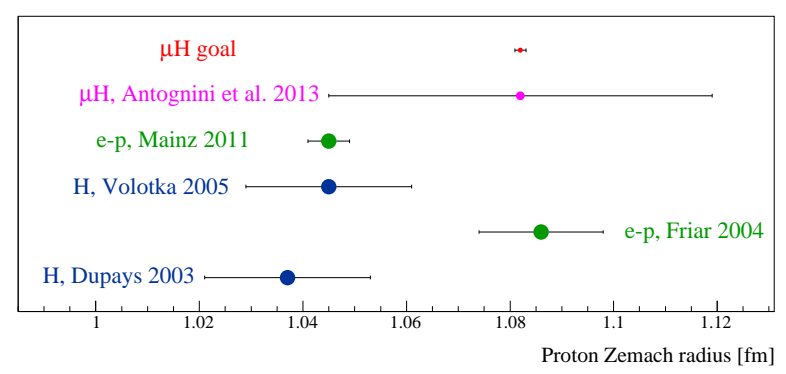

Fig. 6. Proton Zemach radius determinations. Accurate values exist from the $1 \mathrm{~S}$ HFS in hydrogen $(21 \mathrm{~cm}$ line) and elastic electron-proton scattering. The first result from muonic hydrogen will be improved in upcoming experiments.

Previously we presented the determination of the rms charge radii from the measurement of 2S- 
2P Lamb shift transitions in muonic atoms. In a similar way, spectroscopy of the hyperfine splitting (HFS) in muonic and "regular" atoms can be used to deduce precise values of the Zemach radii (of various nuclei). The Zemach radius $R_{Z}$ being defined as an integral of the charge and magnetic form factors $\left(G_{E}, G_{M}\right)$

$$
R_{Z}=-\frac{4}{\pi} \int_{0}^{\infty} \frac{d Q}{Q^{2}}\left(G_{E}\left(Q^{2}\right) \frac{G_{M}\left(Q^{2}\right)}{1+\kappa_{p}}-1\right),
$$

where $\kappa_{p}$ is the proton anomalous magnetic moment and $Q$ the momentum exchange, contains information about the magnetization distribution inside the nucleus. In fact, in a non-relativistic approximation $R_{Z}$ can be expressed, by the convolution between nuclear charge and magnetic distributions $\rho_{E}(r)$ and $\rho_{M}(r)$

$$
R_{Z}=\int d^{3} \mathbf{r}|\mathbf{r}| \int d^{3} \mathbf{r}^{\prime} \rho_{E}\left(\mathbf{r}-\mathbf{r}^{\prime}\right) \rho_{M}\left(\mathbf{r}^{\prime}\right) .
$$

A first, rather crude measurement of the hyperfine splitting in muonic hydrogen has been achieved by the CREMA collaboration in 2013 [10], see Fig. 6. Here, the difference of two 2S-2P Lamb shift transitions was used to determine the 2S-HFS with an accuracy of $\sim 220 \mathrm{ppm}$.

As a next step, the CREMA collaboration is aiming at the measurements of the ground-state HFS in $\mu \mathrm{p}$ and $\left(\mu^{3} \mathrm{He}\right)^{+}$with relative accuracy of about $1 \mathrm{ppm}$. Two other groups, are also planning to measure the 1S-HFS in $\mu$ p so that, differently to the $2 \mathrm{~S}-2 \mathrm{P}$ case, comparison between muonic results will become possible. Having different experimental schemes, these three experiments allow three independent determinations of the Zemach radius. In the following we depict the three methods proposed by the three collaborations to measure the ground-state HFS.

\subsection{CREMA: Diffusion method}
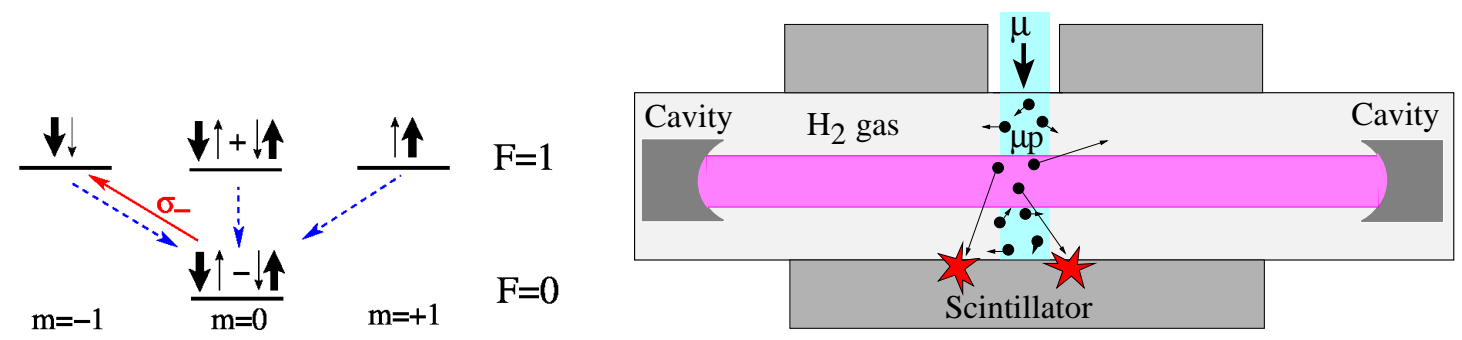

Fig. 7. (Left) Level scheme of the $\mu$ p ground state divided into triplet $(F=1)$ and singlet $(F=0)$ sublevels. The spin composition of the sub-states is also given. The red solid-line arrows represent the transitions driven by the polarized laser light, the dashed blue arrows the quenching of the triplet states into the singlet state caused by collision of the $\mu$ p atoms with the $\mathrm{H}_{2}$ gas. (Right) Principle and setup (not to scale) of the 1S-HFS experiment of the CREMA collaboration. Negative muons stop in the few $\mathrm{mm}$ long gas target at $50 \mathrm{~K}$ and 500 mbar. The $\mu$ p atoms (black dots) excited by the laser light (given in pink) have larger kinetic energy and diffuse from the illuminated volume to the target walls coated with high- $Z$ material. The thermalized $\mu \mathrm{p}$ atoms are much slower. Scintillators are used to observe the cascade events after formation of the excited $(\mu Z)^{*}$ atoms.

The CREMA collaboration experiment is performed at the proton accelerator facility of the Paul Scherrer Institute, Switzerland. A continuous negative muon beam of $10 \mathrm{MeV} / \mathrm{c}$ momentum is stopped in a hydrogen $\left(\mathrm{H}_{2}\right)$ gas target at cryogenic temperatures $(50 \mathrm{~K}, 500 \mathrm{mbar})$. The $\mu^{-}$stopping in the $\mathrm{H}_{2}$ gas forms $\mu$ p atoms in highly excited states $(n \sim 14)$. After the prompt muonic cascade, the $1 \mathrm{~S}$-state is populated with a statistically distributed occupancy of the four sub-levels (triplet and singlet) as visible in Fig. 7 (left). Shortly afterwards, the higher lying triplet states are collisionally quenched to the singlet state (with a rate of $25 / \mu$ s at 1 bar and $273 \mathrm{~K}$ ) [89]), so that at the laser pulse 
arrival time the $\mu \mathrm{p}$ atom is basically in the singlet ground-state. At $1.5 \mu \mathrm{s}$ after the $\mu \mathrm{p}$ formation, the laser system delivers a pulse of $1 \mathrm{~mJ}$ energy at $6.7 \mu \mathrm{m}$ wavelength to drive the hyperfine splitting transition from $F=0$ to $F=1$. Shortly afterwards (within about $100 \mathrm{~ns}$ ), the higher lying triplet state is collisionally quenched back to the singlet state. In this process, the HFS transition energy is converted into kinetic energy: the $\mu \mathrm{p}$ atom gain a kinetic energy of about $120 \mathrm{meV}$. For a sufficiently small target, as shown in Fig. 7 (right) the faster moving $\mu$ p starting from the laser illuminated region may reach the target walls coated with high-Z material before $\mu^{-}$decay occurs [90]. Subsequent $\mu^{-}$ transfer from the $\mu \mathrm{p}$ to a high- $Z$ atom occurs, leading to the formation of a $(\mu Z)^{*}$ atom followed by its de-excitation producing MeV X-rays. Therefore if the laser frequency is resonant with the HFS transition MeV X-rays will be detected right after the laser pulse has illuminated the muon stop distribution. A resonance curve is attained by plotting the number of the cascade signals (from the $\mathrm{MeV}$ $\mathrm{X}$-rays) induced in the surrounding scintillators in a short time window $(\sim 300 \mathrm{~ns})$ after the laser excitation versus the laser frequency.

\subsection{FAMU: Energy dependent muon transfer method}

The methodology of the FAMU collaboration [91,92] differs from the one of the CREMA collaboration mainly in the detection scheme used to expose the laser induced occurrence of the hyperfine transition.

In this experiment which is performed at the RIKEN-RAL muon facility of the Rutherford Laboratory, UK, a pulsed muon beam is stopped in a hydrogen target with 0.045 LHD (liquid hydrogen density). Shortly after its formation the muonic atom ends up in the singlet sub-level of the ground state due to the muonic cascade and the collisional quenching from the triplet to the singlet ground states. A laser pulse is then used to drive the HFS transition, i.e. to excite the muonic atom from the $F=0$ to the $F=1$ state. The atoms excited by the laser in the triplet state, de-excite back to the $F=0$ state through collisions with the $\mathrm{H}_{2}$ molecules gaining in this process about $120 \mathrm{meV}$ of kinetic energy.

The key difference between the CREMA schema and the FAMU scheme is in the method used to distinguish the two classes of atoms: the one which have been laser excited by the laser light, from the one which thermalize at the hydrogen gas temperature. Instead of using the diffusion method of CREMA, the FAMU collaboration utilizes the energy dependence of the muon transfer from the hydrogen atom to a higher- $\mathrm{Z}$ atom which is mixed with a few $\%$ concentration in the $\mathrm{H}_{2}$ gas. The muon transfer rate from the $\mu$ p to some higher-Z gases $\left(\mathrm{CO}_{2}, \mathrm{Ar} ..\right)$ has recently been investigated in a beamtime at the RIKEN-RAL facility [93]. Data analysis is ongoing.

The higher- $Z$ muonic atoms which are formed in highly excited states de-excite down to the ground state producing X-rays in the $10 \mathrm{keV}-1 \mathrm{MeV}$ energy regime that can be efficiently detected with a scintillator or Germanium system. The resonance curve is therefore obtained by plotting the number of X-rays from the "contaminant" gas versus the laser frequency.

\subsection{J-PARC: Decay asymmetry method}

The J-PARC - RIKEN collaboration is planning to use the electron from the muon decay to expose the occurrence of the laser induced transition [94,95]. As mentioned before, after its formation the $\mu \mathrm{p}$ atoms end up quickly in the ground state populating both triplet and singlet states. In this experiment the $\mathrm{H}_{2}$ gas target density is reduced to 0.0001 LHD to minimize the collisional quenching from the triplet into the singlet state. At this density the lifetime of the triplet state is of about $500 \mathrm{~ns}$. The circular polarized laser light which illuminates the target $1 \mu \mathrm{s}$ after the muonic atom formation is inducing a transition from the singlet to a triplet state. This transition which give rise to a muon spin flip, results in a change of the average muon polarization (which is zero prior to the laser pulse). This change of the muon polarization after the laser transition can be exposed by detecting the electron from muon decay. In fact, due to the parity violation in the muon decay, the electron from muon decay 
is emitted preferentially anti-parallel to the muon spin. Therefore the change of the muon polarization induced by the laser light results in a change of the electron distribution which can be observed by using electron detectors. A resonance curve can be obtained by plotting the so called asymmetry (difference in counts between two detectors) as a function of the laser frequency. The change of the electron distribution will be visible in this asymmetry plot.

The choice of the low gas density results from a trade-off between muon stopping volume and efficiency, and the collisional quenching rate from the polarized triplet (after the laser excitation) into the singlet (unpolarized). Therefore the time window where the electrons have to be detected is only of about $1 \mu$ s given the above-mentioned depolarization rate.

\subsection{Impact of the HFS measurements}

By comparing the measured HFS in $\mu \mathrm{p}$ and $\left(\mu^{3} \mathrm{He}\right)^{+}$with the corresponding theoretical predictions $[31,96,97]$ the two-photon-exchange TPE contribution with a relative accuracy of $10^{-4}$ can be extracted. From the TPE the polarizability contribution can be extracted if a Zemach radius is assumed from electron scattering [98,99] or from regular atom spectroscopy [100,101]. Alternatively the Zemach radii can be extracted if the polarizabilities are assumed from theory. A comparison of these radii and polarizability contributions with theory (Chiral perturbation theory [102], dispersion relations [103,104], lattice calculations [105], and ab-initio few nucleon theories [86]) offers the possibility to improve our knowledge of the low energy structure of the of the proton and of one of the simplest nuclei, the helion.

\section{Conclusions}

Laser spectroscopy of muonic atoms can be used for the determination of nuclear parameter with high accuracy, to be compared with the values from electron scattering and regular atom spectroscopy. A large activity both, theoretical and experimental, have been triggered by the observed discrepancy, which remains still unsolved. Many new activities are underway, such as spectroscopy in (regular) atomic hydrogen [106-110], molecular hydrogen (and its isotopes) [111-114], and $\mathrm{He}^{+}$ions [77-83], new elastic electron scattering experiments [115-118], and muon scattering on the proton [119].

\section{Acknowledgments}

We acknowledge support from the European Research Council (ERC StG. 279765), the MaxPlanck-Society and the Max-Planck-Foundation, the Swiss National Science Foundation (project 200020-100632, 200021L_138175, 200020_159755) and the Swiss Academy of Engineering Sciences, the BQR de l'UFR de physique fondamentale et appliquée de l'Université Pierre et Marie Curie- Paris 6, the program PAI Germaine de Staël no. 07819NH du ministère des affaires étrangères France, the Fundação para a Ciência e a Tecnologia (Portugal) and FEDER (project PTDC/FIS/102110/2008 and grants SFRH/BPD/46611/2008, SFRH/BPD/74775/2010, and SFRH/BPD/76842/2011), research centre grant No. UID/FIS/04559/2013 (LIBPhys) from FCT/MCTES/PIDDAC, Portugal, Deutsche Forschungsgemeinschaft (DFG) GR 3172/9-1 within the D-A-CH framework, and Ministry of Science and Technology, Taiwan, No. 100-2112-M-007-006-MY3. P. Indelicato acknowledges support by the "ExtreMe Matter Institute, Helmholtz Alliance HA216/EMMI".

\section{References}

[1] G. Fiorentini and O. Pitzurra: Nuovo Cimento A 43 (1978) 396.

[2] R. O. Mueller, V. W. Hughes, H. Rosenthal, and C. S. Wu: Phys. Rev. A 11 (1975) 1175.

[3] H. P. von Arb, F. Dittus, H. Heeb, H. Hofer, F. Kottmann, S. Niggli, R. Schaeren, D. Taqqu, J. Unternährer, and P. Egelhof: Physics Letters 136B (1984) 232. 
[4] K. Kirch, D. Abbott, P. Bach, B. DeCecco, P. Hauser, D. Horvath, F. Kottmann, J. Missimer, R. T. Siegel, L. M. Simons, and D. Viel: Phys. Rev. Lett. 78 (1997) 4363.

[5] A. Di Giacomo: Nucl. Phys. B 11 (1969) 411.

[6] G. W. F. Drake and L. L. Byer: Phys. Rev. A 32 (1985) 713.

[7] D. F. Measday: Phys. Rep. 354 (2001) 243.

[8] G. Carboni and G. Fiorentini: Nuovo Cimento 39B (1977) 281.

[9] R. Pohl, A. Antognini, F. Nez, F. D. Amaro, F. Biraben, J. M. R. Cardoso, D. S. Covita, A. Dax, S. Dhawan, L. M. P. Fernandes, A. Giesen, T. Graf, T. W. Hänsch, P. Indelicato, L. Julien, C.-Y. Kao, P. Knowles, E.-O. L. Bigot, Y.-W. Liu, J. A. M. Lopes, L. Ludhova, C. M. B. Monteiro, F. Mulhauser, T. Nebel, P. Rabinowitz, J. M. F. dos Santos, L. A. Schaller, K. Schuhmann, C. Schwob, D. Taqqu, J. F. C. A. Veloso, and F. Kottmann: Nature 466 (2010) 213.

[10] A. Antognini, F. Nez, K. Schuhmann, F. D. Amaro, F. Biraben, J. M. R. Cardoso, D. S. Covita, A. Dax, S. Dhawan, M. Diepold, L. M. P. Fernandes, A. Giesen, T. Graf, A. L. Gouvea, T. W. Hänsch, P. Indelicato, L. Julien, C.-Y. Kao, P. Knowles, F. Kottmann, E.-O. L. Bigot, Y.-W. Liu, J. A. M. Lopes, L. Ludhova, C. M. B. Monteiro, F. Mulhauser, T. Nebel, P. Rabinowitz, J. M. F. dos Santos, L. A. Schaller, C. Schwob, D. Taqqu, J. F. C. A. Veloso, J. Vogelsang, and R. Pohl: Science 339 (2013) 417.

[11] R. Pohl, F. Nez, L. M. P. Fernandes, F. D. Amaro, F. Biraben, J. M. R. Cardoso, D. S. Covita, A. Dax, S. Dhawan, M. Diepold, A. Giesen, A. L. Gouvea, T. Graf, T. W. Hänsch, P. Indelicato, L. Julien, P. Knowles, F. Kottmann, E.-O. L. Bigot, Y.-W. Liu, J. A. M. Lopes, L. Ludhova, C. M. B. Monteiro, F. Mulhauser, T. Nebel, P. Rabinowitz, J. M. F. dos Santos, L. A. Schaller, K. Schuhmann, C. Schwob, D. Taqqu, J. F. C. A. Veloso, and A. Antognini: Science 353 (2016) 669.

[12] A. Antognini, F. Nez, F. D. Amaro, F. Biraben, J. M. R. Cardoso, D. S. Covita, A. Dax, S. Dhawan, L. M. P. Fernandes, A. Giesen, T. Graf, T. W. Hänsch, P. Indelicato, L. Julien, C.-Y. Kao, P. Knowles, F. Kottmann, E.-O. L. Bigot, Y.-W. Liu, J. A. M. Lopes, L. Ludhova, C. M. B. Monteiro, F. Mulhauser, T. Nebel, P. Rabinowitz, J. M. F. dos Santos, L. A. Schaller, K. Schuhmann, C. Schwob, D. Taqqu, J. F. C. A. Veloso, and R. Pohl: Can. J. Phys. 89 (2010) 47.

[13] T. Nebel, F. Amaro, A. Antognini, F. Biraben, J. Cardoso, D. Covita, A. Dax, L. Fernandes, A. Gouvea, T. Graf, T. Hänsch, M. Hildebrandt, P. Indelicato, L. Julien, K. Kirch, F. Kottmann, Y.-W. Liu, C. Monteiro, F. Nez, J. Santos, K. Schuhmann, D. Taqqu, J. Veloso, A. Voss, and R. Pohl: Hyp. Interact. 212 (2012) 195

[14] R. Pohl, H. Daniel, F. J. Hartmann, P. Hauser, F. Kottmann, V. E. Markushin, M. Muhlbauer, C. Petitjean, W. Schott, D. Taqqu, and P. Wojciechowski-Grosshauser: Phys. Rev. Lett. 97 (2006) 193402.

[15] A. Antognini, F. D. Amaro, F. Biraben, J. M. R. Cardoso, C. A. N. Conde, D. S. Covita, A. Dax, S. Dhawan, L. M. P. Fernandes, T. W. Hänsch, V. Hughes, O. Huot, P. Indelicato, L. Julien, P. E. Knowles, F. Kottmann, Y.-W. Liu, J. A. M. Lopes, L. Ludhova, C. M. B. Monteiro, F. Mulhauser, F. Nez, B. N. Perry, R. Pohl, P. Rabinowitz, J. M. F. dos Santos, L. A. Schaller, C. Schwob, D. Taqqu, and J. F. C. A. Veloso: Opt. Comm. 253 (2005) 362.

[16] A. Antognini, K. Schuhmann, F. D. Amaro, F. Biraben, A. Dax, A. Giesen, T. Graf, T. W. Hänsch, P. Indelicato, L. Julien, K. Cheng-Yang, P. E. Knowles, F. Kottmann, E. Le Bigot, Y.-W. Liu, L. Ludhova, N. Moschüring, F. Mulhauser, T. Nebel, F. Nez, P. Rabinowitz, C. Schwob, D. Taqqu, and R. Pohl: IEEE J. Quant. Electr. 45 (2009) 993.

[17] L. Fernandes, A. Antognini, M. Boucher, C. Conde, O. Huot, P. Knowles, F. Kottmann, L. Ludhova, F. Mulhauser, R. Pohl, L. Schaller, J. dos Santos, D. Taqqu, and J. Veloso: Nucl. Inst. Meth. A 498 (2003) 362.

[18] L. Ludhova, F. D. Amaro, A. Antognini, F. Biraben, J. M. R. Cardoso, C. A. N. Conde, D. S. Covita, A. Dax, S. Dhawan, L. M. P. Fernandes, T. W. Hänsch, V.-W. Hughes, O. Huot, P. Indelicato, L. Julien, P. E. Knowles, F. Kottmann, J. A. M. Lopes, Y.-W. Liu, C. M. B. Monteiro, F. Mulhauser, F. Nez, R. Pohl, P. Rabinowitz, J. M. F. dos Santos, L. A. Schaller, D. Taqqu, and J. F. C. A. Veloso: Nucl. Inst. Meth. A 540 (2005) 169.

[19] L. M. P. Fernandes, F. D. Amaro, A. Antognini, J. M. R. Cardoso, C. A. N. Conde, O. Huot, P. E. Knowles, F. Kottmann, J. A. M. Lopes, L. Ludhova, C. M. B. Monteiro, F. Mulhauser, R. Pohl, J. M. F. dos Santos, L. A. Schaller, D. Taqqu, and J. F. C. A. Veloso: Journal of Instrumentation 2 (2007) P08005.

[20] J. L. Friar: Phys. Rev. C 16 (1977) 1540.

[21] J. L. Friar: Ann. Phys. 122 (1979) 151. 
[22] E. Borie and G. A. Rinker: Rev. Mod. Phys. 54 (1982) 67.

[23] R. Pohl, A. Antognini, F. Amaro, F. Biraben, J. Cardoso, C. Conde, A. Dax, S. Dhawan, L. Fernandes, T. Hänsch, F. Hartmann, V. Hughes, O. Huot, P. Indelicato, L. Julien, P. Knowles, F. Kottmann, Y.-W. Liu, L. Ludhova, C. Monteiro, F. Mulhauser, F. Nez, P. Rabinowitz, J. dos Santos, L. Schaller, C. Schwob, D. Taqqu, and J. Veloso: Can. J. Phys. 83 (2005) 339.

[24] T. Nebel, F. D. Amaro, A. Antognini, F. Biraben, J. M. R. Cardoso, C. A. N. Conde, A. Dax, S. Dhawan, L. M. P. Fernandes, A. Giesen, T. W. Hänsch, P. Indelicato, L. Julien, P. E. Knowles, F. Kottmann, E. Le Bigot, Y.-W. Liu, J. A. M. Lopes, L. Ludhova, C. M. B. Monteiro, F. Mulhauser, F. Nez, R. Pohl, P. Rabinowitz, J. M. P. dos Santos, L. A. Schaller, K. Schuhmann, C. Schwob, D. Taqqu, and J. F. C. A. Veloso: Can. J. Phys. 85 (2007) 469.

[25] K. Pachucki: Phys. Rev. A 53 (1996) 2092.

[26] K. Pachucki: Phys. Rev. A 60 (1999) 3593.

[27] E. Borie: Phys. Rev. A 71 (2005) 032508.

[28] A. P. Martynenko: Phys. Rev. A 71 (2005) 022506.

[29] A. P. Martynenko: Phys. At. Nucl. 71 (2008) 125.

[30] M. I. Eides, H. Grotch, and V. A. Shelyuto: Phys. Rep. 342 (2001) 63 .

[31] M. I. Eides, H. Grotch, and V. A. Shelyuto: Theory of Light Hydrogenlike Atoms (Springer Tracts in Modern Physics 222, 2006).

[32] U. D. Jentschura: Ann. Phys. 326 (2011) 500.

[33] U. D. Jentschura: Phys. Rev. A 84 (2011) 012505.

[34] S. G. Karshenboim, V. G. Ivanov, and E. Y. Korzinin: Phys. Rev. A 85 (2012) 032509.

[35] E. Borie: Ann. Phys. 327 (2012) 733.

[36] P. Indelicato: Phys. Rev. A 87 (2013) 022501.

[37] S. G. Karshenboim, E. Y. Korzinin, V. A. Shelyuto, and V. G. Ivanov: Phys. Rev. D 91 (2015) 073003.

[38] A. Antognini, F. Kottmann, F. Biraben, P. Indelicato, F. Nez, and R. Pohl: Ann. Phys. 331 (2013) 127.

[39] P. J. Mohr, B. N. Taylor, and D. B. Newell: Rev. Mod. Phys. 84 (2012) 1527.

[40] R. Pohl, R. Gilman, G. A. Miller, and K. Pachucki: Ann. Rev. Nucl. Part. Sci. 63 (2013) 175.

[41] C. E. Carlson: Prog. Part. Nucl. Phys. 82 (2015) 59.

[42] R. Pohl, F. Nez, T. Udem, A. Antognini, A. Beyer, H. Fleurbaey, A. Grinin, T. W. Hänsch, L. Julien, F. Kottmann, J. J. Krauth, L. Maisenbacher, A. Matveev, and F. Biraben: arXiv 1607.03165 [physics.atom-ph] (2016).

[43] J. C. Bernauer, P. Aschenbach, C. Ayerbe Gayoso, R. Böhm, D. Bosnar, L. Debenjak, M. O. Distler, L. Doria, A. Esser, et al.: Phys. Rev. Lett. 105 (2010) 242001.

[44] I. Sick: Phys. Lett. B 576 (2003) 62.

[45] I. Sick: Few-Body Syst. 55 (2014) 903.

[46] I. Sick and D. Trautmann: Phys. Rev. C 89 (2014) 012201(R).

[47] J. Arrington and I. Sick: J. Phys. Chem. Ref. Data 44 (2015) 031204.

[48] M. Belushkin, H.-W. Hammer, and U.-G. Meissner: Phys. Rev. C 75 (2007) 035202.

[49] I. T. Lorenz, H.-W. Hammer, and U.-G. Meissner: Eur. Phys. J. A 48 (2012) 151.

[50] I. T. Lorenz, U.-G. Meissner, H.-W. Hammer, and Y. B. Dong: Phys. Rev. D 91 (2015) 014023.

[51] K. Griffioen, C. Carlson, and S. Maddox: Phys. Rev. C 93 (2016) 065207.

[52] D. W. Higinbotham, A. A. Kabir, V. Lin, D. Meekins, B. Norum, and B. Sawatzky: Phys. Rev. C 93 (2016) 055207.

[53] M. Horbatsch and E. A. Hessels: Phys. Rev. C 93 (2016) 015204.

[54] E. Borie: Phys. Rev. A 72 (2005) 0522511.

[55] A. A. Krutov and A. P. Martynenko: Phys. Rev. A 84 (2011) 052514.

[56] R. N. Faustov, A. P. Martynenko, G. A. Martynenko, and V. V. Sorokin: Phys. Rev. A 90 (2014) 012520.

[57] R. N. Faustov, A. P. Martynenko, G. A. Martynenko, and V. V. Sorokin: Phys. Rev. A 92 (2015) 052512.

[58] K. Pachucki: Phys. Rev. Lett. 106 (2011) 193007.

[59] J. L. Friar: Phys. Rev. C 88 (2013) 034003.

[60] O. J. Hernandez, C. Ji, S. Bacca, N. Nevo Dinur, and N. Barnea: Phys. Lett. B 736 (2014) 344.

[61] C. E. Carlson, M. Gorchtein, and M. Vanderhaeghen: Phys. Rev. A 89 (2014) 022504.

[62] K. Pachucki and A. Wienczek: Phys. Rev. A 91 (2015) 040503(R). 
[63] J. J. Krauth, M. Diepold, B. Franke, A. Antognini, F. Kottmann, and R. Pohl: Ann. Phys. 366 (2016) 168.

[64] C. G. Parthey, A. Matveev, J. Alnis, R. Pohl, T. Udem, U. D. Jentschura, N. Kolachevsky, and T. W. Hänsch: Phys. Rev. Lett. 104 (2010) 233001.

[65] U. D. Jentschura, A. Matveev, C. G. Parthey, J. Alnis, R. Pohl, T. Udem, N. Kolachevksy, and T. W. Hänsch: Phys. Rev. A 83 (2011) 042505.

[66] S. G. Karshenboim, V. G. Ivanov, E. Y. Korzinin, and V. A. Shelyuto: Phys. Rev. A 81 (2010) 060501(R).

[67] S. G. Karshenboim, E. Y. Korzinin, V. G. Ivanov, and V. A. Shelyuto: JETP Lett. 92 (2010) 8.

[68] A. Krutov, A. Martynenko, G. Martynenko, and R. Faustov: J. Exp. Theo. Phys. 120 (2015) 73.

[69] E. Y. Korzinin, V. G. Ivanov, and S. G. Karshenboim: Phys. Rev. D 88 (2013) 125019.

[70] U. D. Jentschura: Phys. Rev. A 84 (2011) 012505.

[71] U. D. Jentschura and B. J. Wundt: Eur. Phys. J. D 65 (2011) 357.

[72] E. N. Elekina, K. A. A., and M. A. P.: Phys. Part. At. Nucl. 8 (2011) 331.

[73] C. Ji, N. Nevo Dinur, S. Bacca, and N. Barnea: Phys. Rev. Lett. 111 (2013) 143402.

[74] M. Diepold, J. J. Krauth, B. Franke, A. Antognini, F. Kottmann, and R. Pohl: arXiv 1606.05231 [atomph] (2016).

[75] I. Sick: Phys. Rev. C 77 (2008) 041302.

[76] I. Sick: J. Phys. Chem. Ref. Data 44 (2015) 031213.

[77] R. van Rooij, J. S. Borbely, J. Simonet, M. D. Hoogerland, K. S. E. Eikema, R. A. Rozendaal, and W. Vassen: Science 333 (2011) 196.

[78] P. Cancio Pastor, L. Consolino, G. Giusfredi, P. De Natale, M. Inguscio, V. A. Yerokhin, and K. Pachucki: Phys. Rev. Lett. 108 (2012) 143001.

[79] D. Shiner, R. Dixson, and V. Vedantham: Phys. Rev. 74 (1995) 3553.

[80] K. Pachucki, V. A. Yerokhin, and P. Cancio Pastor: Phys. Rev. A 85 (2012) 042517.

[81] M. Herrmann, M. Haas, U. Jentschura, F. Kottmann, D. Leibfried, G. Saathoff, C. Gohle, A. Ozawa, V. Batteiger, S. Knünz, N. Kolachevsky, H. Schüssler, T. Hänsch, and T. Udem: Phys. Rev. A 79 (2009) 052505.

[82] D. Z. Kandula, C. Gohle, T. J. Pinkert, W. Ubachs, and K. S. E. Eikema: Phys. Rev. A 84 (2011) 062512.

[83] J. Morgenweg, I. Barmes, and K. S. E. Eikema: Nature Physics 30 (2014).

[84] E. Epelbaum, H.-W. Hammer, and U.-G. Meißner: Rev. Mod. Phys. 81 (2009) 1773.

[85] Z.-T. Lu, P. Mueller, G. W. F. Drake, W. Nörtershäuser, S. C. Pieper, and Z.-C. Yan: Rev. Mod. Phys. 85 (2013) 1383.

[86] C. Ji, N. Nevo Dinur, S. Bacca, and N. Barnea: Few-Body Syst. 55 (2014) 917.

[87] G. Hagen, A. Ekström, C. Forssén, G. R. Jansen, W. Nazarewicz, T. Papenbrock, K. A. Wendt, S. Bacca, N. Barnea, B. Carlsson, C. Drischler, K. Hebeler, M. Hjorth-Jensen, M. Miorelli, G. Orlandini, A. Schwenk, and J. Simonis.: Nature Physics 12 (2016) 186190.

[88] U. D. Jentschura, A. Czarnecki, and K. Pachucki: Phys. Rev. A 72 (2005) 062102.

[89] J. S. Cohen: Phys. Rev. A 43 (1991) 4668.

[90] D. J. Abbott, G. F. Chen, P. Guss, A. D. Hancock, J. B. Kraiman, R. T. Siegel, W. F. Vulcan, D. W. Viel, R. E. Welsh, C. Petitjean, A. Zehnder, W. H. Breunlich, M. Cargnelli, P. Kammel, A. Scrinzi, J. Marton, J. Zmeskal, J. J. Reidy, H. L. Woolverton, F. J. Hartmann, A. Adamczak, V. E. Markushin, and V. S. Melezhik: Phys. Rev. A 55 (1997) 214.

[91] D. Bakalov, A. Adamczak, M. Stoilov, and A. Vacchi: Hyp. Interact. 233 (2015) 97.

[92] D. Bakalov, A. Adamczak, M. Stoilov, and A. Vacchi: Phys. Lett. A 379 (2015) 151.

[93] A. Adamczak, G. Baccolo, D. Bakalov, G. Baldazzi, R. Bertoni, M. Bonesini, V. Bonvicini, R. Campana, R. Carbone, T. Cervi, F. Chignoli, M. Clemenza, L. Colace, A. Curioni, M. Danailov, P. Danev, I. D’Antone, A. De Bari, C. De Vecchi, M. De Vincenzi, M. Furini, F. Fuschino, K. Gadedjisso-Tossou, D. Guffanti, A. Iaciofano, K. Ishida, D. Iugovaz, C. Labanti, V. Maggi, A. Margotti, M. Marisaldi, R. Mazza, S. Meneghini, A. Menegolli, E. Mocchiutti, M. Moretti, G. Morgante, R. Narda, M. Nastasi, J. Niemela, E. Previtali, R. Ramponi, A. Rachevski, L. P. Rignanese, M. Rossella, P. L. Rossi, F. Somma, M. Stoilov, L. Stoychev, A. Tomaselli, L. Tortora, A. Vacchi, E. Vallazza, G. Zampa, and M. Zuffa: Journal of Instrumentation 11 (2016) P05007.

[94] M. Sato, K. Ishida, M. Iwasaki, S. Kanda, Y. Ma, Y. Matsuda, T. Matsuzaki, K. Midorikawa, Y. Oishi, S. Okada, N. Saito, K. Tanaka, and S. Wada: Proceedings of the 20th International Conference on Particles and Nuclei (PANIC 14), 24-29 Aug 2014. Hamburg, Germany - (2015) 460. 
[95] N. Saito, M. Yumoto, T. Tomida, U. Takagi, and S. Wada: Proc. SPIE 8526 (2012) 852605.

[96] S. G. Karshenboim: Phys. Rep. 422 (2005) 1.

[97] A. P. Martynenko: J. Exp. Theo. Phys. 106 (2008) 690.

[98] M. O. Distler, J. C. Bernauer, and T. Walcher: Phys. Lett. B 696 (2011) 343.

[99] I. Sick: Phys. Rev. C 90 (2014) 064002.

[100] A. Dupays, A. Beswick, B. Lepetit, C. Rizzo, and D. Bakalov: Phys. Rev. A 68 (2003) 052503.

[101] A. V. Volotka, V. M. Shabaev, G. Plunien, and G. Soff: Eur. Phys. J. D 33 (2005) 23.

[102] F. Hagelstein, R. Miskimen, and V. Pascalutsa: Prog. Part. Nucl. Phys. 88 (2016) 29.

[103] C. E. Carlson, V. Nazaryan, and K. Griffioen: Phys. Rev. A 78 (2008) 022517.

[104] C. E. Carlson, V. Nazaryan, and K. Griffioen: Phys. Rev. A 83 (2011) 042509.

[105] C. Alexandrou, M. Constantinou, G. Koutsou, and M. P. K. Ottnad: arXiv:1605.07327 (2016).

[106] A. C. Vutha, B. N., I. Ferchichi, M. C. George, V. Isaac, C. H. Storry, A. S. Weatherbee, W. Weel, and E. A. Hessels: Bull. Am. Phys. Soc. 57(5) (2012) Q1.138.

[107] A. Beyer, J. Alnis, K. Khabarova, A. Matveev, C. G. Parthey, D. C. Yost, R. Pohl, T. Udem, T. W. Hänsch, and N. Kolachevsky: Ann. d. Phys. (Berlin) 525 (2013) 671.

[108] A. Beyer, C. G. Parthey, N. Kolachevsky, J. Alnis, K. Khabarova, R. Pohl, E. Peters, D. C. Yost, A. Matveev, K. Predehl, S. Droste, T. Wilken, R. Holzwarth, T. W. Hänsch, M. Abgrall, D. Rovera, C. Salomon, P. Laurent, and T. Udem: J. Phys. Conf. Ser. 467 (2013) 012003.

[109] S. Galtier, H. Fleurbaey, S. Thomas, L. Julien, F. Biraben, and F. Nez: J. Phys. Chem. Ref. Data 44 (2015) 031201.

[110] D. C. Yost, A. Matveev, A. Grinin, E. Peters, L. Maisenbacher, A. Beyer, R. Pohl, N. Kolachevsky, K. Khabarova, T. W. Hänsch, and T. Udem: Phys. Rev. A 93 (2016) 042509.

[111] S. Schiller, D. Bakalov, and V. I. Korobov: Phys. Rev. Lett. 113 (2014) 023004.

[112] G. D. Dickenson, M. L. Niu, E. J. Salumbides, J. Komasa, K. S. E. Eikema, K. Pachucki, and W. Ubachs: Phys. Rev. Lett. 110 (2013) 193601.

[113] J. Biesheuvel, J.-P. Karr, L. Hilico, K. S. E. Eikema, W. Ubachs, and J. C. J. Koelemeij: Nature Comm. 7 (2015) 10385.

[114] J. Karr, L. Hilico, J. Koelemeij, and V. Korobov: arXiv 1605.05456 (physics.atom-ph) (2016).

[115] M. Mihovilovic and H. Merkel: AIP Conf. Proc. 1563 (2013) 187.

[116] M. Meziane: AIP Conf. Proc. 1563 (2013) 183.

[117] A. Gasparian: EPJ Web Conf. 73 (2014) 07006.

[118] Y. Kohl: PoS Bormio2015 (2015) 005.

[119] R. Gilman: AIP Conf. Proc. 1563 (2013) 167. 